\title{
Review
}

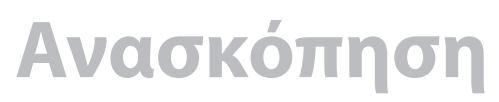

\section{Dietary interventions and cognition: A systematic review of clinical trials}

\author{
V. Gkotzamanis, ${ }^{1}$ D. Panagiotakos ${ }^{1,2}$ \\ 'Department of Nutrition and Dietetics, School of Health Science and Education, Harokopio University, Athens, Greece, \\ ${ }^{2}$ Faculty of Health, University of Canberra, Canberra, Australia
}

Psychiatriki 2020, 31:248-256

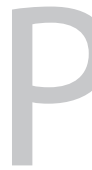

revalence of Alzheimer's Disease and other forms of dementia is increasing in accordance with the increase of life expectancy and the resulting world population aging, while an effective pharmaceutical treatment is pending. These facts underline the need for development of targeted interventions that could decrease the incidence of dementia. Dietary supplementation, especially sources of $\omega-3$ fatty acids and polyphenols such as fish oil and blueberries respectively, have been reported to have a beneficial effect on cognitive functioning. The aim of this review is to summarize the most recent findings of clinical studies investigating the effect of dietary supplementation on cognitive performance and identify potential effective interventions. For this purpose, PubMed, Scopus and Google Scholar research was conducted and a total of ten studies met the selection criteria. Four of these studies investigated the effect of $\omega-3$ fatty acid supplementation. Two of these presented significant benefits in certain domains of cognitive functions (such as working memory, space imagery efficiency perceptual speed), in full scale IQ as well as prevention of hippocampal atrophy while the remaining two did not report any improvements. Two more studies investigated the effect of polyphenol supplementation and reported minor benefits in spatial memory as well as enhanced stimulation of certain brain regions. One study compared the effect of fish oil and blueberry supplementation as well as their combination and presented cognitive benefits for both fish oil and blueberries but not for their simultaneous administration. Finally, three more studies investigated the effect of DW 2009 soybean, ashwagandha and a nutraceutical formulation and reported cognitive benefits in attention, memory and global cognition respectively for their intervention groups. In total, eight studies investigated interventions on people with Mild Cognitive Impairment or Subjective Cognitive Impairment and all of them reported significant cognitive benefits in some cognitive domains. On the contrary, the remaining two studies included individuals with diagnosed dementia reported minimal to hardly any benefits. Conclusively, the interventions of the studies reviewed seem promising for individuals at risk of dementia, but not for those who are already diagnosed with dementia. However, further research is required to validate their effect as well as determine recommended doses.
\end{abstract}

Key words: Diet, nutrition, dietary supplements, cognition, Alzheimer disease, dementia. 


\section{Introduction}

Cognitive impairment in the form of Alzheimer's Disease (AD) and other types of dementia, is the main contributor to disability in elderly creating a great burden for both patients and their caregivers. ${ }^{1,2}$ Mild Cognitive Impairment (MCl) is defined as a condition between dementia and the expected cognitive decline of normal aging. ${ }^{3}$ Subjective Cognitive Impairment (SCl) is defined as the state of self-experience of deterioration in cognitive performance which cannot be detected by objective neuropsychological tests. ${ }^{4}$ Both these conditions $(\mathrm{MCl}$ and $\mathrm{SCl})$ are associated with elevated risk of progressing to dementia later in life. As there is currently no effective pharmaceutical treatment, there is an imperative need for targeted interventions that could decrease the burden of dementia, especially in the stages preceding it, such as $\mathrm{MCl}$ and $\mathrm{SCl}$, as once it is clinically diagnosed there is little prospect for improvement in $A D .^{5}$

Dietary interventions have been studied as a means of preserving cognitive performance in high-risk individuals. Particularly, w3-fatty acids and polyphenols are associated in many studies with improvement of cognitive function, however they are not the only dietary interventions that are reported to benefit cognitive performance. Docosahexaenoic acid (DHA) and Eicosipentaenoic acid (EPA) are $\omega-3$ fatty acids contained in fish oil. DHA is the main component of the neuronal cell membranes. As a result, it takes part in the cell's ionic exchange and interacts directly with membrane proteins, thus, regulates cells' communication. In addition, DHA and EPA, have been shown in animal models to decrease levels of $A \beta$ amyloid, a molecule that plays a key role in $A D{ }^{6,7}$ What is more, DHA and EPA have been shown to promote anti-inflammatory molecules (leukotrienes, resolvins, neuroprotection D1) ${ }^{8}$ as well as reduce brain oxidative stress in animal models. ${ }^{9}$ As a result, they are believed to have a major role in regulating inflammation, another key aspect of the pathogenesis of AD. All the mechanisms mentioned above, as well as their proved beneficial effect on cardiovascular system ${ }^{10}$ make DHA and EPA a promising intervention for the protection of cognitive function.

Polyphenols, particularly anthocyanins, contained mostly in blueberries, are also believed to have neuroprotective properties. ${ }^{11}$ Animal models have shown that blueberry intake may improve age-related cognitive decline in various aspects of cognition. Blueberry supplementation is reported to reduce markers of inflammation and oxidative stress as well as upregulate neurogenesis, neuroplasticity and brain-derived neurotrophic factor. ${ }^{12}$ Furthermore, they are reported to benefit cardiovascular health via their anti-inflammatory properties, thus further benefiting brain tissues by improving their blood perfusion. ${ }^{13,14}$ As a conclusion, blueberry supplementation can also be considered a promising dietary intervention for the protection of cognitive functioning.

The aim of this systematic review was to summarize and synthesize data from the latest randomized trials investigating the effect of dietary interventions on cognitive functions and the population that is expected to have the most benefit from these interventions.

\section{Material and method \\ Literature selection criteria}

The inclusion criteria for this review were the following: The studies had to be randomized placebo controlled clinical trials, published in English but with no limitation regarding the country where they were conducted, published in the past five years.

\section{Outcome measures}

The outcomes of interest were all the different domains of cognitive functioning measured by various neuropsychological tests.

\section{Search strategy}

A literature search on PubMed, Scopus and Google Scholar, was conducted. The search query included the following terms: "diet", "dietary supplementation" "cognitive function", "cognition", "cognitive impairment". Additional methodological filters were also used: publication in the past 5 years, studies in humans only, and clinical trial design. Figure 1 summarizes the search strategy that was followed.

\section{Data extraction}

At first, papers were manually checked based on the Title and Abstract screening and then full text reading for the final selection decision. 


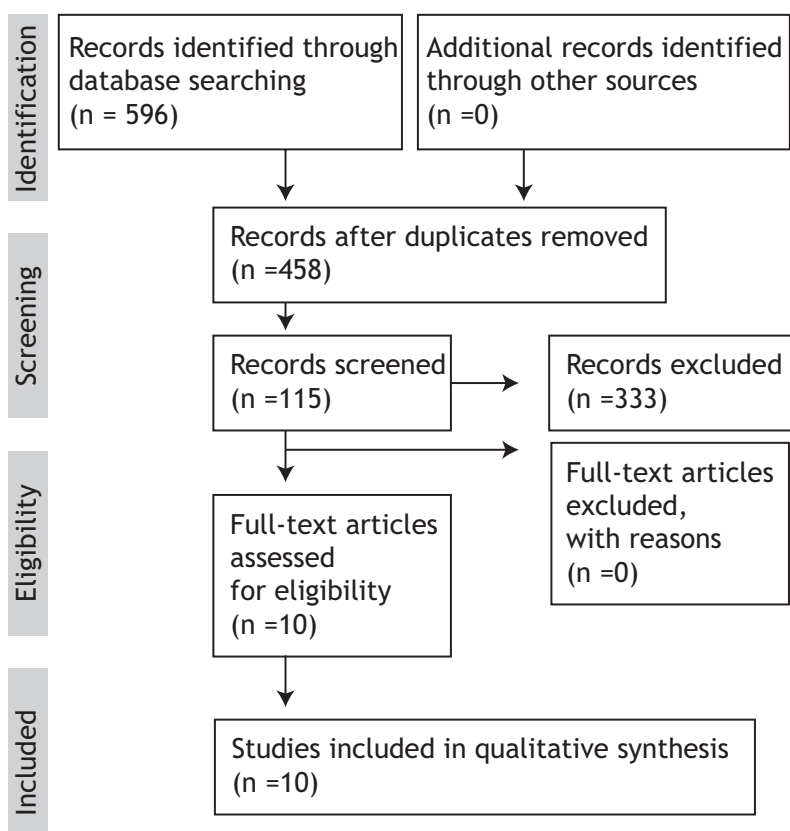

Figure 1. PRISMA Diagram of search strategy for the review of intervention trials of dietary supplementation on cognitive performance.

\section{Quality assessment}

Risk of bias for each study was estimated using the Cochrane Rob 2 tool (table 1).

\section{Findings of the studies}

The search query provided 596 results. After Title and Abstract screening and then full text reading a total of ten studies met all the criteria mentioned above. Table 2 summarizes the characteristics of the studies that were selected

Based on the literature review of the ten studies that met the inclusion criteria, it seems that certain nutrients from fish and berries have significant effects on cognitive function. In particular, Zhang et $\mathrm{al}^{15}$ conducted a double blind RCT in order to investigate the effect of DHA supplementation on cognitive function and hippocampal volume in 240 community-living individuals, aged over 65 , with $\mathrm{MCl}$. The participants were randomized into two groups and were administered either a daily dose of $2 \mathrm{mg}$ of an algal-derived DHA or identical placebo. Cognitive outcomes included the Chinese version of Wechsler Adult Intelligence Scale Revised
Table 1. Risk of bias estimated with Cochrane Rob 2 tool.

\begin{tabular}{lll}
\hline Year & Study & $\begin{array}{c}\text { Estimated risk } \\
\text { of bias }\end{array}$ \\
\hline 2017 & Boespflug EL & High Risk \\
2016 & Hashimoto M & High Risk \\
2016 & Zhang YP & Low Risk \\
2016 & Lamport DJ & Low Risk \\
2017 & Choudhary D & Low Risk \\
2017 & Bo Y & Low Risk \\
2019 & Hwang YH & Low Risk \\
2015 & Remington R & Low Risk \\
2017 & Mcnamara RK & Some Concerns \\
2015 & Eriksdotter M & Some Concerns \\
\hline
\end{tabular}

(WAIS-RC) and hippocampal volume measured via MRI. Age-appropriate norms from the Chinese standardization were used for the calculation of the Intelligence Quotient (IQ) and index scores. The results showed significant benefits for the intervention group (IG) compared to the control group (CG) in Full Scale IQ as well as in some subcategories of WAISRC. Furthermore, total volume of hippocampus increased for IG while it decreased for CG.

In another double-blind randomized trial, Bo et al ${ }^{16}$ investigated the effect of $n-3$ polysaturated fatty acids (PUFAs) supplementation on cognitive function in 86 Chinese elders with $\mathrm{MCl}$. The participants were randomized into two groups and received either a daily dose of $480 \mathrm{mg}$ DHA and $720 \mathrm{mg}$ of EPA in a capsule form or isocaloric capsules of placebo (olive oil) respectively. Cognitive functioning was assessed with Basic Cognitive Aptitude Tests (BCATs). The results showed that the intervention group had significant improvement in their BCATs' scores $(p<0.001)$. In particular, perceptual speed, space imagery efficiency and working memory were significantly improved $(p<0.05)$.

Eriksdotter et $\mathrm{al}^{17}$ investigated the effect of a 6-month administration of $\omega$-3 fatty acid supplementation on the cognitive function of 174 patients with $A D$ in relation to their lipidaemic profiler. Participants were randomized to receive four capsules daily each containing either $430 \mathrm{mg}$ of DHA and $150 \mathrm{mg}$ of EPA or placebo). Cognitive outcomes included Mini 


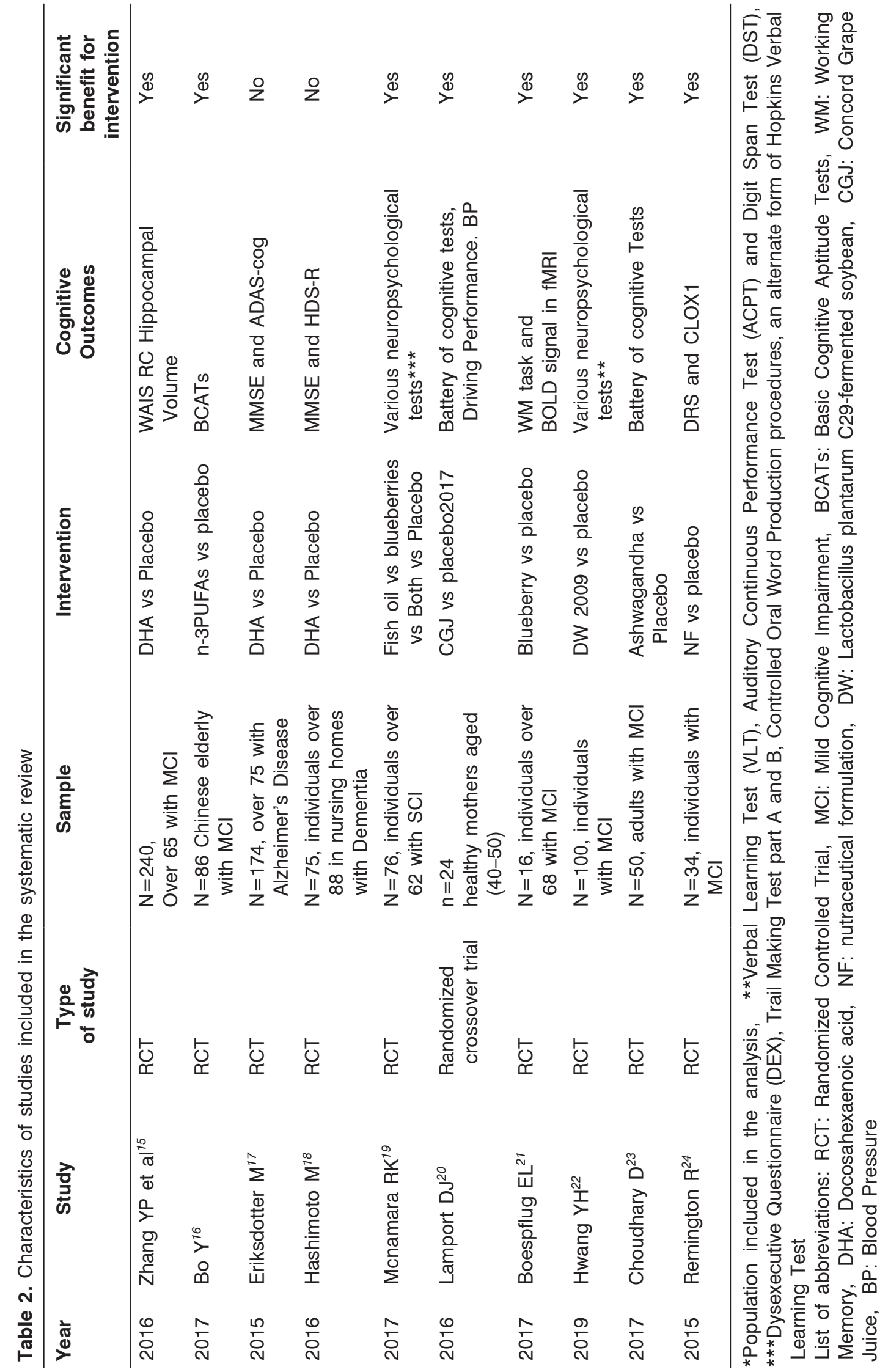


Mental State Examination (MMSE) and Alzheimer's Disease Assessment Scale (ADAS-cog). The results showed a significant positive association between changes in plasma DHA levels and ADAS-cog total scores $(p=0.016)$ No association was found between changes in plasma fatty acid profile and MMSE score. In addition, there was no association at baseline between gender and fatty acid profile or severity of dementia and fatty acid profile.

In a single blind randomized interventional study Hashimoto et $\mathrm{al}^{18}$ investigated the effect of DHA intervention on cognitive functioning and mental health of 75 Japanese elderly individuals (aged over 88) living in care facilities and nursing homes. Participants were randomized in two groups and received $1720 \mathrm{mg}$ dose of DHA or placebo. Cognitive outcomes included MMSE score and Dementia ScaleRevised (HDS-R). The results showed no significant difference in the total scores of the tests. However, when test subitems were analyzed the mean changes in subitem "Registration" of MMSE at six and twelve months was significantly higher in the intervention group $(p=0.01)$. Moreover, total MMSE score was positively correlated with DHA levels $(r=0.247$, $\mathrm{p}=0.031$ ).

Conducting a 24-week randomized, double-blind, placebo-controlled trial Mcnamara et al ${ }^{19}$ investigated the effect of fish oil, blueberry and combined supplementation on cognitive functioning of 94 adults with $\mathrm{SCl}$, aged 62-80. Participants were randomized into four groups: FO (fish oil+placebo powder, $n=21$ ), $B B$ (blueberry powder+placebo oil, $n=24), F O+B B$ (fish oil+ blueberry powder, $\mathrm{n}=26$ ) and PL (placebo oil+ placebo powder, $n=23$ ). Cognitive outcomes included the Dysexecutive Questionnaire (DEX), Trail Making Test part A and B, Controlled Oral Word Production procedures to evaluate lexical access under constraint, as well as alternate form of Hopkins Verbal Learning Test. The results showed that FO groups $(p=0.03)$ and BB groups (0.05) reported significantly fewer cognitive symptoms. In addition, the BB groups showed improved memory discrimination $(p=0.04)$.

In a randomized crossover trial that was conducted by Lamport et $\mathrm{al}^{20}$ the effect of Concord grape juice (CGJ), a rich source of polyphenols, was investigated for its effect on cognitive functioning and driving performance of 25 British mothers of preteens, aged 40-50. They were randomized into one of two crossovers (CGJ then placebo or placebo then CGJ) and consumed daily $355 \mathrm{~mL}$ of CGJ or placebo. The cognitive outcomes included a 45-min cognitive test battery which comprised 7 subtests. The results showed a significant improvement in immediate spatial memory and in driving performance, following the CGJ intervention.

In another double-blind, randomized, placebocontrolled trial Boespflug et $\mathrm{al}^{21}$ investigated the effect of blueberry supplementation on neural activation and working memory in 16 individuals with $\mathrm{MCl}$ aged over 68. Participants were randomized into two groups of eight and received twice daily either $12.5 \mathrm{~g}$ of blueberry powder or identical placebo. The outcome of this study was a working memory task with a simultaneous record of Blood Oxygen LevelDependent (BOLD) signal in $\mathrm{FMRI}$ while the participants performed this test. The results showed no difference in the performance of the two groups. However, BOLD signal indicated increased activation in certain regions during the working memory task in the blueberry treated group $(p<0.01)$.

In a double-blind, mutli-centre, placebo-controlled clinical trial, Hwang et $\mathrm{a}^{22}$ investigated the effect of Lactobacillus plantarum C29-fermented soybean (DW2009), a source of isoflavones, on the cognitive function of 100 individuals with $\mathrm{MCl}$, aged $55-85$. Participants were randomized to receive either a daily dose of $800 \mathrm{mg}$ of DW2009 in the form of a capsule or indistinguishable placebo. Cognitive outcomes Verbal Learning Test (VLT), Auditory Continuous Performance Test (ACPT) and Digit Span Test (DST). The results showed significantly greater improvement in the combined cognitive function for the intervention group compared to placebo, especially in the attention domain composite score.

Conducting a prospective, randomized, double blind, placebo-controlled trial, Choudhary et $\mathrm{al}^{23}$ investigated the effect of ashwagandha, a herb used in the Indian Ayurvedic system, on cognitive function of 50 adults with $\mathrm{MCl}$. The participants were randomized into two groups and were administered twice daily either a dose of $300 \mathrm{mg}$ of ashwagandha root extract or identical placebo. Cognitive outcomes included tests assessing memory (immediate 
memory, general memory, working memory), visuospatial processing and response, executive function, and attention capabilities. The results showed significant improvements for the ashwagandha treatment compared to placebo in immediate and general memory, executive function as well as in attention and information processing speed, while they were inconclusive for the working memory index.

In a randomized clinical trial Remington et $\mathrm{al}^{24}$ investigated the effect of a nutraceutical formulation (NF) containing folate, alpha-tocopherol, vitamin B12, S-adenosyl methionine, $\mathrm{N}$-acetyl cysteine and acetyl-L-carnitine on the cognitive functioning of 34 people with $\mathrm{MCl}$. Participants were randomized to receive either NF in the form of a tablet or indistinguishable placebo. Cognitive function was assessed with Dementia Rating Scale (DRS) and CLOX-1. The results showed that participants who received NF had a significant increase in their DRS results in three months and maintained this level of improvement for the duration of the study. After the intervention period there was and open label extension during which placebo group switched to NF. In this period the placebo group presented significant improvement (Cohen's Effect Size $=0.35$ ).

\section{Discussion}

\section{S-3 fatty acids}

Four studies investigated the effect of $\omega-3$ fatty acid supplementation on the cognitive functioning compared to a placebo. The results of these studies seemed to vary depending on their population. Specifically, the two studies that included patients with dementia did not report a substantial improvement of cognitive function compared to their control groups. On the contrary, the studies that included patients with $\mathrm{MCl}$ reported significant improvements for their intervention groups compared to their control groups in their primary outcomes, WAIS-RC and BCATs respectively. These findings may indicate that once dementia and especially $A D$ have been diagnosed little can be done to reverse their progress.

\section{Polyphenol supplementation}

Two studies investigated the effect of polyphenol supplementation, in the form of concord grape juice and blueberries, on the cognitive function of healthy mothers and patients with $\mathrm{MCl}$ respectively. Lamport et al with a crossover trial, reported significant benefits in certain categories of the battery tests that were used as primary outcome, as well as in the additional driving task, between the intervention and the control group. Although this study is limited by its small size $(n=24)$, it makes an interesting implication that healthy individuals may also benefit by its intervention. On the other hand, Boespflug et al did not report any benefit in the performance of the WM task, however the intervention altered significantly BOLD signal in $\mathrm{fMRI}$. Although these findings do not show a beneficial effect on cognitive function, they suggest that the intervention may increase stimulation of certain brain regions providing some interesting insights in mechanisms that may be further researched.

\section{Blueberry versus fish oil}

One trial investigated the effect of blueberry and fish oil supplementation as well as their combination. This study included participants over 62 years old with $\mathrm{SCl}$. This study reported cognitive benefits for both the fish oil and the blueberry. Furthermore, the blueberry group also reported improved memory discrimination at the end of the intervention period. Surprisingly, the combined group, that received both fish oil and blueberry supplementation, not only did it not present a higher accumulative effect, but it actually showed no cognitive benefit. A potential explanation provided by the researchers, suggests that the reason for this surprising finding lies in the common molecular path that both $\omega$-3 fatty acids and flavonoids follow. Specifically, both interventions' effects are suggested to be mediated by the transcription factor NF-E-2 related factor 2 (Nrf2). Although acute activation of Nrf2 is believed to have an anti-inflammatory effect, prolonged and excessive upregulation may have the opposite effect.

\section{Ashwagandha, DW and NF}

Three more studies reported the effect of interventions that do not belong in the categories of $\omega-3$ fatty acids or polyphenols. All these studies included participants with $\mathrm{MCl}$ and based their hypothesis on previous studies. Ashwagandha is a herb that has long been used in the Ayurvedic system to improve cognition. DW2009 is a soybean fermented with C29 
lactobacillus plantarum that has been shown to have cognitive enhancing and anti-inflammatory properties in animal models. NF is a nutraceutical formulation containing folate, alpha tocopherol, B12, Sadenosyl methionine, $\mathrm{N}$-acetylcysteine and acetyl-Lcarnitine, that has been shown to improve cognition in patients with AD. All these interventions showed significant cognitive benefits for their intervention groups. Although their mechanisms are not clarified, an anti-inflammatory action is common is ground for them. Moreover, DW2009 is reported to alter gut microbiota, which could be another potential mechanism given the rising number of studies that report a link between gut microbiota and various neurodegenerative diseases. Their findings may seem prom- ising, however there is still need for further research and validation as the data supporting their effect in published literature is far less compared to $\omega-3$ fatty acids and flavonoids.

\section{Conclusions}

$\Omega-3$ fatty acids and flavonoid rich foods such as blueberries are consistently reported to have a significant protective role in cognitive functioning, when administered to people at risk but not with a clinical diagnosis of dementia. In addition, more interventions, such as ashwagandha, DW2009 and NF, look promising, but there is still need for further research and validation.

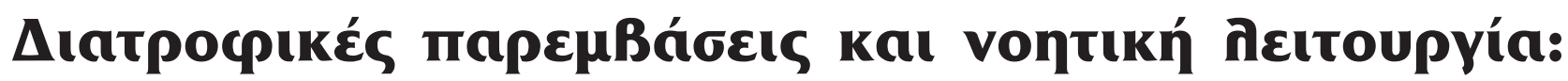

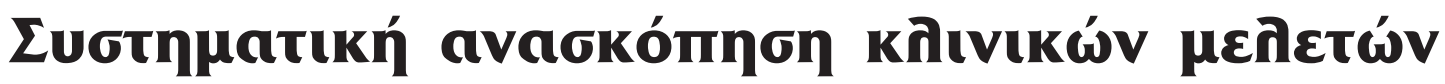

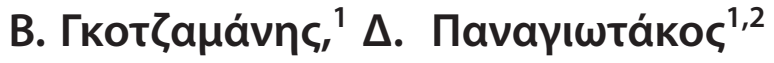

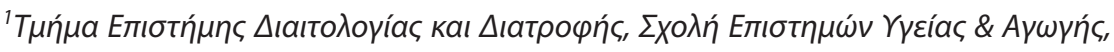

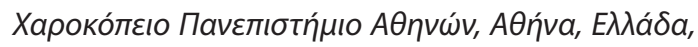

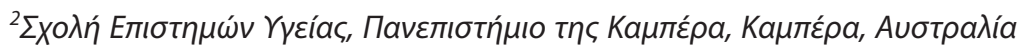

Uuxıатрıкń 2020, 31:248-256

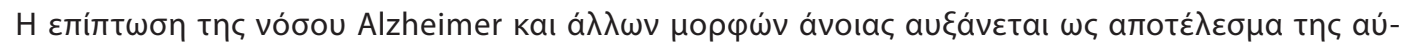

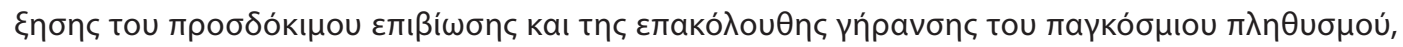

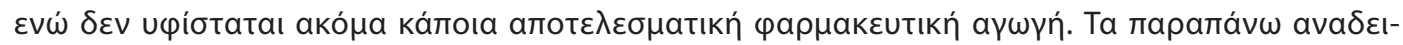

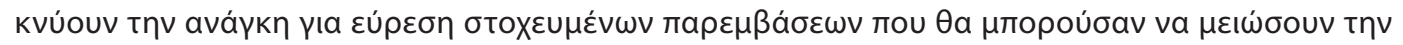

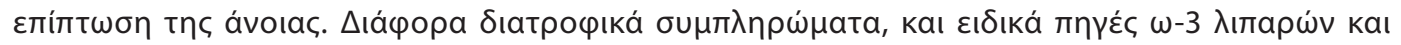

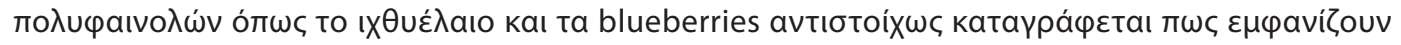

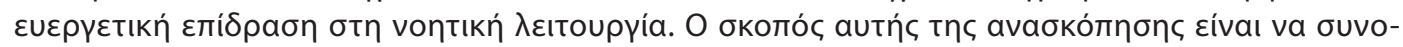

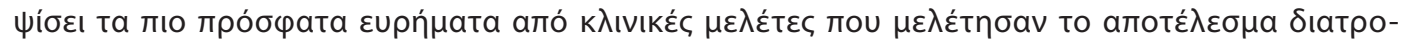

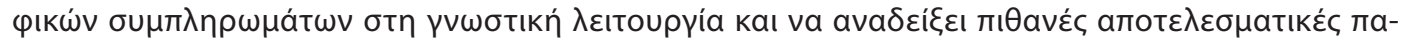

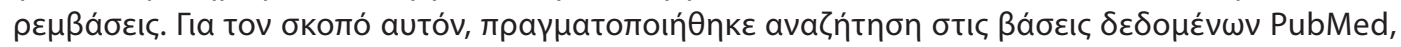

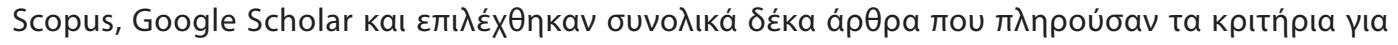

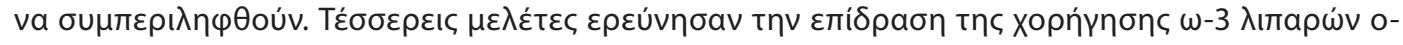

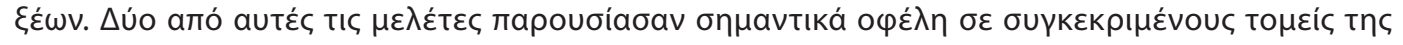

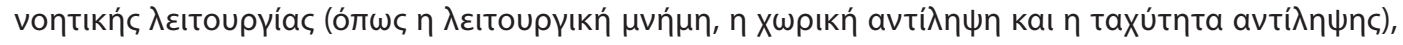

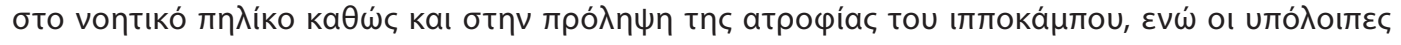

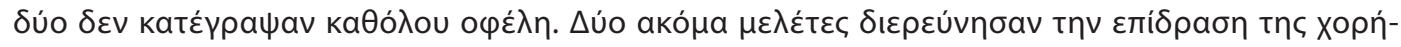

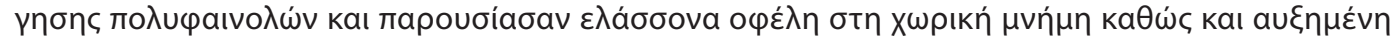

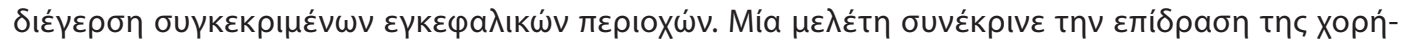




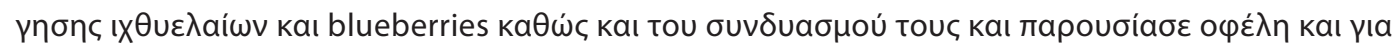

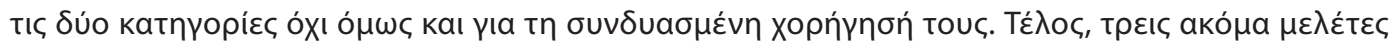

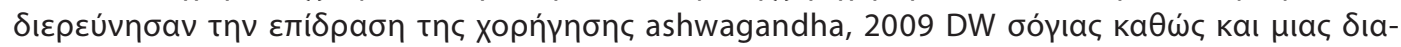

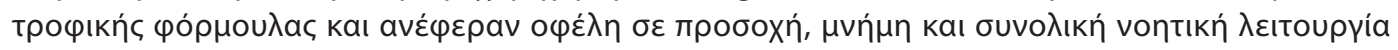

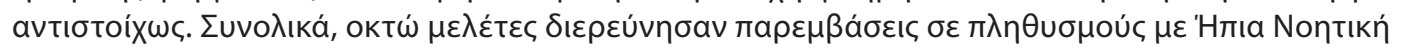

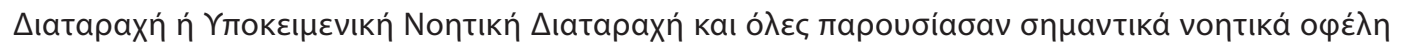

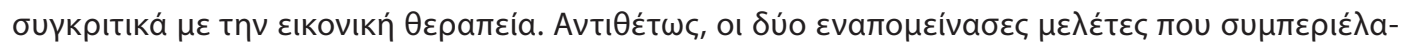

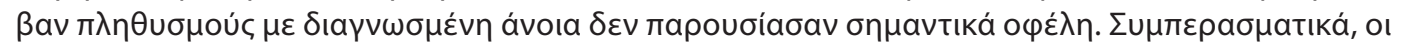

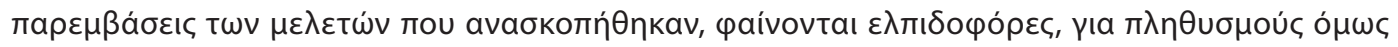

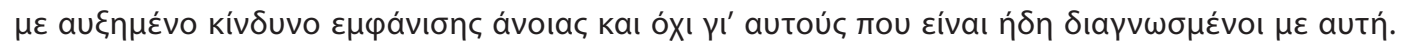

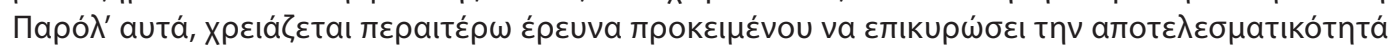

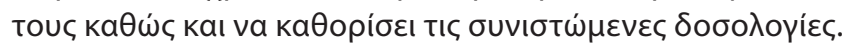

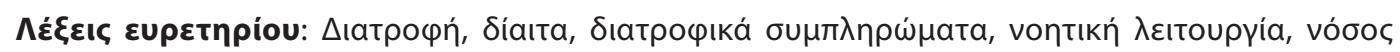
Alzheimer, ávoı́a.

\section{References}

1. Wajman JR, Mansur LL, Yassuda MS. Lifestyle Patterns as a Modifiable Risk Factor for Late-life Cognitive Decline: A Narrative Review Regarding Dementia Prevention. Curr Aging Sci 2018, 11:90-99, doi: 10.2174/1874609811666181003160225

2. Mougias AA, Politis A, Mougias MA, Kotrotsou I, Skapinakis $P$, Damigos D, Mavreas VG. The burden of caring for patients with dementia and its predictors. Psychiatriki 2015, 26:28-37, PMID: 25880381

3. Ronald C. Petersen. Mild Cognitive Impairment Continuum 2016, 22(2 Dementia):404-18, doi: 10.1212/CON.0000000000000313

4. Studart A Neto, Nitrini R. Subjective cognitive decline: The first clinical manifestation of Alzheimer's disease? Dement Neuropsychol 2016, 10:170-177, doi: 10.1590/S1980-5764-2016 DN1003002

5. Blennow K, de Leon MJ, Zetterberg H. Alzheimer's disease. Lancet 2006, 368:387-403, doi: 10.1016/S0140-6736(06)69113-7

6. Cunnane SC, Plourde M, Pifferi F, Bigin M, Fiart C, BarbergerGateau P. Fish, docosahexaenoic acid and Alzheimer's disease. Prog Lipid Res 2009, 48:239-256, doi: 10.1016/j.plipres.2009.04.001

7. Calon F, Cole G. Neuroprotective action of omega-3 polyunsaturated fatty acids against neurodegenerative diseases: evidence from animal studies. Prostaglandins Leukot Essent Fatty Acids 2007, 77:287-293, doi: 10.1016/j.plefa.2007.10.019

8. Solfrizzi V, D'Introno A, Colacicco AM, Capurso C, Todarello $\mathrm{O}$, Pellicani V. Circulating biomarkers of cognitive decline and dementia. Clin Chim Acta 2006, 364:91-112, doi: 10.1016/j.cca. 2005.06.015

9. Cole GM, Lim GP, Yang F, Teter B, Begum A, Ma Q. Prevention of Alzheimer's disease: Omega-3 fatty acid and phenolic antioxidant interventions. Neurobiol Aging 2005, 26(Suppl 1):133136, doi: 10.1016/j.neurobiolaging.2005.09.005

10. Balk EM, Horsley TA, Newberry SJ, Lichtenstein AH, Yetley EA, Schachter HM. A collaborative effort to apply the evidencebased review process to the field of nutrition: challenges, ben- efits, and lessons learned. Am J Clin Nutr 2007, 85:1448-1456, doi: 10.1093/ajcn/85.6.1448

11. Kalt W, Cassidy A, Howard LR, Krikorian R, Stull AJ, Tremblay F et al. Recent Research on the Health Benefits of Blueberries and Their Anthocyanins. Adv Nutr 2020, 11:224-236, doi: 10.1093/ advances/nmz065

12. Casadesus G, Shukitt-Hale B, Stellwagen HM, Zhu X, Lee HG, Smith MA et al. Modulation of hippocampal plasticity and cognitive behavior by short-term blueberry supplementation in aged rats. Nutr Neurosci 2004, 7:309-16, doi: 10.1080/10284150400020482

13. Grosso G, Micek A, Godos J, Pajak A, Sciacca S, Galvano $F$ et al. Dietary Flavonoid and Lignan Intake and Mortality in Prospective Cohort Studies: Systematic Review and DoseResponse Meta-Analysis. Am J Epidemiol 2017, 185:1304-1316, doi: 10.1093/aje/kww207

14. Wang X, Ouyang YY, Liu J, Zhao G. Flavonoid intake and risk of CVD: a systematic review and meta-analysis of prospective cohort studies. Br J Nutr 2014, 111:1-11, doi: 10.1017/ S000711451300278X

15. Zhang YP, Miao R, Li Q, Wu T, Ma F. Effects of DHA Supplementation on Hippocampal Volume and Cognitive Function in Older Adults with Mild Cognitive Impairment: A 12-Month Randomized, Double-Blind, Placebo-Controlled Trial. J Alzheimers Dis 2017, 55:497-507, doi: 10.3233/JAD-160439

16. Bo Y, Zhang X, Wang Y, You J, Cui H, Zhu Y. The n-3 polyunsaturated Fatty Acids Supplementation Improved the Cognitive Function in the Chinese Elderly with Mild Cognitive Impairment: A Double-Blind Randomized Controlled Trial. Nutrients 2017, 9:54, doi: 10.3390/nu9010054, doi: 10.3390/nu9010054

17. Eriksdotter M, Vedin I, Falahati F, Freund-Levi Y, Hjorth R, FaxenIrving G. Plasma Fatty Acid Profiles in Relation to Cognition and Gender in Alzheimer's Disease Patients During Oral Omega-3 Fatty Acid Supplementation: The OmegAD Study. J Alzheimers Dis 2015, 48:805-812, doi: 10.3233/JAD-150102 
18. Hashimoto M, Kato S, Tanabe Y, Katakura M, Mamun AA, Ohno $M$. Beneficial effects of dietary docosahexaenoic acid intervention on cognitive function and mental health of the oldest elderly in Japanese care facilities and nursing homes. Geriatr Gerontol Int 2017, 17:330-337, doi: 10.1111/ggi.12691

19. McNamara RK, Kalt W, Shidler MD, McDonald J, Summer SS, Stein AL. Cognitive response to fish oil, blueberry, and combined supplementation in older adults with subjective cognitive impairment. Neurobiol Aging 2018, 64:147-156, doi: 10.1016/j. neurobiolaging.2017.12.003

20. Lamport DJ, Lawton CL, Merat N, Jamson H, Myrissa K, Hofman $\mathrm{D}$ et al. Concord grape juice, cognitive function, and driving performance: a 12-wk, placebo-controlled, randomized crossover trial in mothers of preteen children. Am J Clin Nutr 2016, 103:775-783, doi: 10.3945/ajcn.115.114553

21. Boespflug EL, Eliassen JC, Dudley JA, Shidler MD, Kalt W, Summer SS. Enhanced neural activation with blueberry supplementation in mild cognitive impairment. Nutr Neurosci 2018, 21:297-305, doi: 10.1080/1028415X.2017.1287833

22. Hwang YH, Park S, Paik JW, Chae SW, Kim DH, Jeong DG. Efficacy and Safety of Lactobacillus Plantarum C29-Fermented Soybean (DW2009) in Individuals with Mild Cognitive Impairment: A 12-Week, Multi-Center, Randomized, DoubleBlind, Placebo-Controlled Clinical Trial. Nutrients 2019, 11: 305, doi: 10.3390/nu11020305
23. Choudhary D, Battacharyya S, Bose S. Efficacy and Safety of Ashwagandha (Withania somnifera (L.) Dunal) Root Extract in Improving Memory and Cognitive Functions. J Diet Supp/ 2017, 14:6, 599-612, doi: 10.1080/19390211.2017.1284970

24. Remington R, Lortieb JJ, Hoffmann H, Page R, Morrell C, Shea TB. A Nutritional Formulation for Cognitive Performance in Mild Cognitive Impairment: A Placebo-Controlled Trial with an Open-Label Extension. J Alzheimers Dis 2015, 48:591-595, doi: $10.3233 / J A D-150057$

Corresponding author: Demosthenes Panagiotakos, Harokopio University, 70 El. Venizelou street, GR-176 71 Athens, Greece, Tel: (+30) 2109549332

e-mail: dbpanag@hua.gr 